\title{
Evodiamine inhibits the proliferation of human osteosarcoma cells by blocking PI3K/Akt signaling
}

\author{
ZI-JUN MENG ${ }^{1}$, NIAN WU ${ }^{1}$, YANG LIU ${ }^{1}$, KE-JIE SHU ${ }^{1}$, XIANG ZOU ${ }^{1}$, RAN-XI ZHANG ${ }^{1}$, CHANG-JUN PI $^{1}$, \\ BAI-CHENG HE ${ }^{2}$, ZHEN-YONG KE ${ }^{1}$, LIANG CHEN ${ }^{1}$, ZHONG-LIANG DENG ${ }^{1}$ and LIANG-JUN YIN ${ }^{1}$ \\ ${ }^{1}$ Department of Orthopedic Surgery, The Second Affiliated Hospital, Chongqing Medical University, Chongqing; \\ ${ }^{2}$ Pharmacy School of Chongqing Medical University, Chongqing, P.R. China
}

Received March 11, 2015; Accepted May 4, 2015

DOI: $10.3892 /$ or.2015.4084

\begin{abstract}
Osteosarcoma (OS) is the most common non-hematologic primary malignancy of bone, and multiple chemotherapeutic agents have been applied in the treatment of OS for over 40 years. Nevertheless, due to the poor prognosis of OS, it is essential to develop a novel treatment strategy. Evodiamine (EVO), a quinolone alkaloid extracted from the fruit of Evodia rutaecarpa, has been demonstrated to inhibit tumor cell proliferation. Thus, the main aim of the present study was to investigate the anti-proliferative and apoptosis-inducing effects of evodiamine (EVO) on human OS 143B cells, but also the possible mechanisms underlying these effects. The results of crystal violet staining, flow cytometry, western blot analysis and an in vivo experiment demonstrated that EVO exhibits significant inhibitory effects on cell proliferation, exhibits apoptosis-inducing effects and arrests the cell cycle in 143B cells. According to our findings of polymerase chain reaction (PCR), western blot analysis and recombinant adenoviral transfection, we confirmed that EVO upregulates both the protein and gene levels of phosphatase and tensin homolog (PTEN) in a concentration-dependent manner in 143B cells. Overexpression of PTEN reinforced the anti-proliferative effect of EVO in the 143B cells, while knockdown of PTEN upregulated PI3K/Akt signaling transduction and reversed the inhibitory effect of EVO on 143B cell proliferation. Further analysis indicated that EVO upregulated the expression of PTEN by inactivating PI3K/Akt signaling by decreasing phosphorylated Akt1/2. Based on the above results, we conclude that PTEN/PI3K/Akt signaling is involved in the inhibitory effect on human OS 143B cell proliferation by EVO.
\end{abstract}

Correspondence to: Professor Liang-Jun Yin, Department of Orthopedic Surgery, The Second Affiliated Hospital, Chongqing Medical University, 76 Linjiang Road, Yuzhong, Chongqing 400016, P.R. China

E-mail: albertyin.21@163.com

Key words: evodiamine, osteosarcoma, proliferation inhibition, PTEN/PI3K/Akt

\section{Introduction}

The most common non-hematologic primary malignancy of bone, osteosarcoma (OS), mainly occurs in children and adolescents $(1,2)$. In fact, OS is the third most common malignancy in adolescents and accounts for over $56 \%$ of all bone sarcomas. OS is a primary sarcoma of adolescents, but it is believed to present as secondary neoplasms attributed to malignant transformation of Paget disease in older individuals (3-5). OS is often located in the metaphysis of long bones, and the most common sites have been reported to be linked to rapid bone growth in the younger age group, including the distal femur, proximal tibia and proximal humerus $(4,6,7)$. Due to the application of pre-operative and post-operative multiagent chemotherapy combined with gradually developed surgical techniques for OS management, the long-term survival rate for localized OS has improved to $\sim 60 \%$ (8), still, $33 \%$ of OS patients present with recurrent disease and OS patients rarely achieve recovery status (9). OS patients commonly succumb to respiratory system failure due to the highly pulmonary metastatic feature of OS (4). Therefore, more efficient antitumor drugs for OS are urgently needed. Recently, traditional Chinese medicine (TCM) has received increased attention. A high curative effect has been noted when combining TCM and chemotherapy agents for various types of cancers.

Evodiamine (EVO; 8,13,13b,14-tetrahydro-14-methylindolo[2'3'-3,4]pyrido[2,1-b]quinazolin-5-[7H]-one), a quinolone alkaloid isolated from a Chinese herbal medicinal plant Evodia rutaecarpa (10), plays multiple roles in biological physiological process, such as anti-inflammatory (11), anti-nociceptive (12) and uterotonic effects (13). Currently, evidence indicates that EVO possesses anticancer activities. EVO is reported to hinder tumor development by inhibiting cancer cell proliferation and inducing cell apoptosis in various types of cancer cells, such as lung (14), acute leukemia (15-17), prostate (18-20) and cervical (17). Yang et al reported that EVO can upregulate the expression of phosphatase shatterproof 1 (SHP-1) (21), which is the key gene of IL-6-induced signal transducer and activator of transcription signaling 3 (STAT3) signaling leading to the suppression of survival and proliferation in hepatocellular carcinoma cells. Yet, little is known concerning the possible molecular mechanisms underlying the EVO-induced apoptosis of OS cells. 
Phosphatidylinositol 3 kinase (PI3K)/Akt signaling dominates many aspects of cell growth, cell apoptosis and cell cycle progression (22). The hyperactivated PI3K/Akt pathway has been identified as the most pivotal impetus in tumor development (23). Although the components of PI3K/Akt signaling are regulated by a range of factors, phosphatase and tensin homologue (PTEN), considered as an anti-oncogene (24), is the only known lipid phosphatase attenuating PI3K signaling transduction (23). Mutations of PTEN occur frequently in many types of carcinomas, such as prostate cancer (25) and human glioma (26). PTEN serves as a phosphatase for the lipid-signaling second messenger phosphatidylinositol3,4,5-trisphosphate (PIP3) (27). The 3'-phosphate on PIP3 is hydrolyzed to generate PIP2 by PTEN, thereby, PTEN can directly antagonize PI3K/Akt signaling transduction (28).

In the present study, we investigated the possible mechanisms involved in the anti-proliferative and apoptosis-inducing effects of EVO in OS cells. Our data demonstrated that EVO inhibits the proliferation of OS cells and the antitumor effect of EVO is mediated by attenuating the PI3K/Akt signaling pathway through downregulation of the expression of PTEN.

\section{Materials and methods}

Cell culture and agents. The human OS cell line 143B was purchased from the American Type Culture Collection (ATCC) and EVO was obtained from Hao-xuan Bio-tech Co., Ltd. (Xi'an, China). EVO was dissolved in dimethyl sulfoxide (DMSO) for the in vitro experiment. VO-OHpic was purchased from Sigma-Aldrich (St. Louis, MO, USA). Antibodies were obtained from Santa Cruz Biotechnology (Santa Cruz, CA, USA). EVO was prepared with $0.4 \%$ carboxymethylcellulose sodium (CMC-Na) as suspension for the in vivo experiments. All other reagents were purchased from Sigma-Aldrich or Fisher Scientific, unless otherwise indicated. Cells were maintained in Dulbecco's modified Eagle's medium (DMEM) with $10 \%$ fetal bovine serum (FBS), $100 \mathrm{U} / \mathrm{ml}$ of penicillin and $100 \mu \mathrm{g} / \mathrm{ml}$ of streptomycin at $37^{\circ} \mathrm{C}$ in $5 \% \mathrm{CO}_{2}$.

Viability assay of the cell cultures. Cell viability was determined using the crystal violet assay, and it was conducted as previously described (29). In brief, 143B cells were plated in a 24-well plate and treated with predesigned concentrations of EVO. The cells were washed carefully with $4^{\circ} \mathrm{C}$ phosphatebuffered saline (PBS) and stained with $0.5 \%$ crystal violet formalin solution to assess the cell viability at room temperature for 20-30 min. For quantification and imaging, a $1 \mathrm{ml}$ aliquot of $20 \%$ acetic acid was added to the well to dissolve the crystal violet and the plate was shaken for $20 \mathrm{~min}$ at room temperature. The absorbance was estimated at $570 \mathrm{~nm}$. Each test was conducted in triplicate.

Flow cytometric analysis of cell cycle distribution and apoptosis. The cells were plated into 6-well plates. For cell cycle analysis, the cells were treated with the indicated concentrations of EVO or DMSO for $48 \mathrm{~h}$. Then, the cells were washed with PBS $\left(4^{\circ} \mathrm{C}\right)$, washed and collected with cold $70 \%$ ethanol $\left(4^{\circ} \mathrm{C}\right)$ followed by washing with $50 \%$ and $30 \%$ ethanol and PBS. After the above operation, the cells were incubated with $1 \mathrm{ml}$ of $20 \mathrm{mg} / \mathrm{ml}$ propidium iodide (PI) which contained
RNase $(1 \mathrm{mg} / \mathrm{ml})$ in PBS for $30 \mathrm{~min}$ followed by fluorescenceactivated cell sorting (FACS) assay. For the apoptosis assay, the cells were collected after treatment with the indicated concentrations of EVO for $48 \mathrm{~h}$. The cells were washed with cold $\left(4^{\circ} \mathrm{C}\right) \mathrm{PBS}$, followed by incubation with PI and Annexin V-EGFP according to the kit procedures (KeyGen Biotech Co. Ltd., Nanjing, China). Then, the processed cells were inspected using FACS assay.

Construction of the recombinant adenoviruses. Recombinant adenoviruses expressing PTEN (Ad-PTEN), GFP (Ad-GFP) and small interfering RNA (siRNA) fragments transfection were performed for the detection of PTEN (Ad-siPTEN). All these recombinant adenovirus were generated previously using the AdEasy technology, as described (30).

Reverse transcription and polymerase chain reaction analysis (RT-PCR). Human OS 143B cells were seeded in T25 flasks and treated with the indicated concentrations of EVO or solvent for 24 and $48 \mathrm{~h}$. Total RNA was extracted using TRIzol reagent (Invitrogen, Carlsbad, CA, USA) and used to obtain cDNA templates by RT reaction. Then, the cDNAs were used as templates for determining the expression of target genes by PCR. The primers were: GAPDH forward, 5'-CAACGAATTTGGCTACAGCA-3' and reverse, 5'-AGGGGAGATTCAGTGTGGTG-3'; PTEN forward, 5'-TAAAGGCACAAGAGGCCCTA-3' and reverse, 5'-CGC CACTGAACATTGGAATA-3'.

Western blot analysis. Subconfluent 143B cells were plated in 6 -well plates and treated with the indicated concentrations of EVO or DMSO. At the pre-designed time points ( 24 and $48 \mathrm{~h}$ ), the cells were washed with cold $\left(4^{\circ} \mathrm{C}\right) \mathrm{PBS}$ followed by being lysed with $300 \mu 1$ lysis buffer. Then, the lysates were boiled for $10 \mathrm{~min}$. Extracted total proteins were separated by SDS-PAGE and then transferred onto polyvinylidene difluoride (PVDF) membranes. Bovine serum albumin (BSA) (10\%) was used to block the membranes at room temperature for $1 \mathrm{~h}$, and then the membranes were blotted with primary antibodies. Finally, the images of the target proteins were detected with SuperSignal West Pico Chemiluminescent substrate.

Xenograft tumor model of human osteosarcoma. All animal experiments abided by the guidelines of the Institutional Animal Care and Use Committee of Chongqing Medical University (IACUC, Chongqing, China) and were approved by the IACUC. Athymic nude mice (female, 4- to 6-weeks old, 5/group) were obtained from the Animal Center of Chongqing Medical University (Chongqing, China). The cells (143B) were washed and resuspended in cold $\left(4^{\circ} \mathrm{C}\right) \mathrm{PBS}$. Then the cells were injected into the backs of nude mice by subcutaneous injection $\left(2.5 \times 10^{7}\right.$ cells/injection). Three days after injection, the athymic nude mice were treated with different doses of EVO (20 and $50 \mathrm{mg} / \mathrm{kg}$ ) or solvent by intragastric administration once a day. After a 4-week intragastric administration, the mice were sacrificed and the sarcoma samples were retrieved for histological evaluation.

Immunohistochemical staining and histological evaluation. Formulin (10\%) was used to stain the retrieved tumor samples 


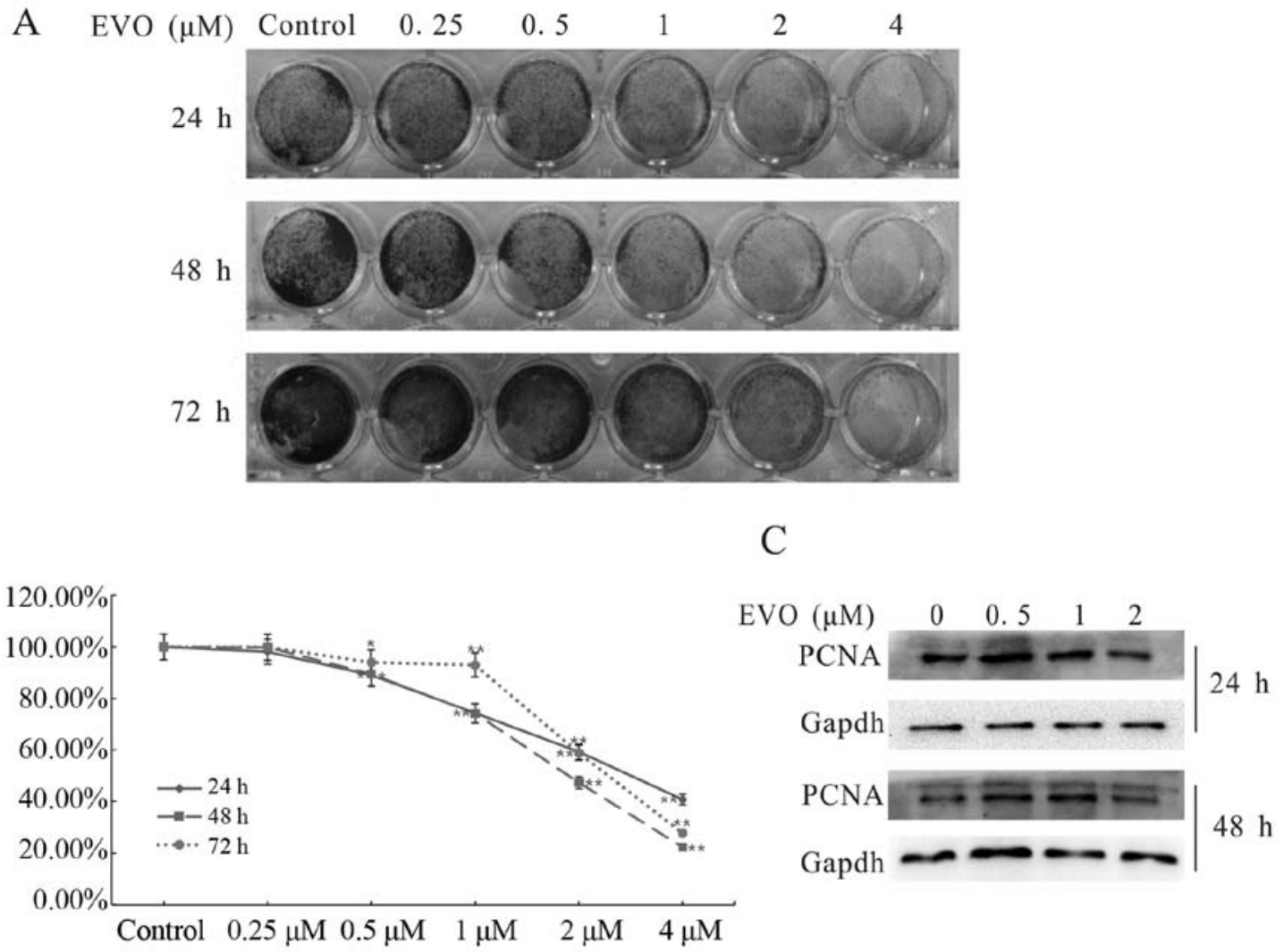

Figure 1. The inhibitory effect of EVO on OS cell proliferation. (A) Crystal violet staining results in the 143B cells. Subconfluent 143B cells were seeded in 24-well plates and treated with different concentrations of EVO or solvent, followed by crystal violet staining at the indicated time-points. (B) Quantitative results of the crystal violet staining in 143B cells. The quantification of crystal violet staining results was processed as described in Materials and methods. The assay was performed in triplicate. "P $<0.05$, compared with the control; ${ }^{* *} \mathrm{P}<0.01$, compared with the control. (C) Western blot analysis results indicating the PCNA protein level in the 143B cells. GAPDH was used as the loading control. OS, osteosarcoma; EVO, evodiamine.

and paraffin was used to embed the slides respectively (31). For the immunohistochemical staining, the processed slides were deparaffinized and then rehydrated in a graduated manner. The deparaffinized sections were subjected to antigen retrieval. Then the background was blocked and the slides were incubation with a primary anti-proliferating cell nuclear antigen (PCNA) antibody, or mouse IgG as the control group. Finaly, the primary PCNA was contrasted by 3,3'-diaminobezidine (DAB) staining (31).

Statistical analysis. All the experiments were performed at least twice independently and the results were repeated in triplicate. The data are represented as mean \pm standard deviation. Statistics from Microsoft Excel software were analyzed using the Student's t-test among different groups. A $\mathrm{P}<0.05$ was judged to be statistically significant.

\section{Results}

Evodiamine inhibits the proliferation of human osteosarcoma cells. Previous research has reported the antitumor effect of EVO on digestive system tumors. To explore whether EVO has the potential to be a novel pharmacotherapy for OS patients, we used crystal violet staining to evaluate the inhibitory effect of EVO on OS cell proliferation. The results revealed that EVO inhibited the proliferation of 143B cells in a concentration- and time-dependent manner (Fig. 1A and B). As shown in Fig. 1C, the activity of PCNA, which plays a key role in the cell cycle (32), was obviously suppressed. Data clearly indicated that EVO is capable of inhibiting the proliferation of $143 \mathrm{~B}$ cells.

Evodiamine induces apoptosis in human osteosarcoma cells. We aimed to explore whether EVO induces apoptosis in 143B cells. We first employed FACS and western blot analysis to estimate the effect of EVO on the apoptosis induction in 143B cells. The results demonstrated that EVO induced the apoptosis of the $143 \mathrm{~B}$ cells in a time- and concentrationdependent manner (Fig. 2A and B). For further investigation, Annexin V-EGFP staining was performed. Cells (143B) were treated with the indicated concentrations of EVO for $24 \mathrm{~h}$ followed by staining with Annexin V-EGFP fusion protein. The results demonstrated that EVO induced the apoptosis of 143B cells in a concentration-dependent manner (Fig. 2C). These results strongly indicate EVO can induce apoptosis in human OS 143B cells.

Evodiamine upregulates the expression of PTEN and arrests the cell cycle at $G 1$ phase. PTEN has been proven to possess antitumor activity in many carcinomas for its vital role in the inhibition of cell proliferation $(33,34)$. Therefore, we investigated whether PTEN exhibited anti-proliferative activity in 143B cells. The experimental results of the PCR and western blot analyses (Fig. 3B and C) demonstrated that EVO concentration 
A
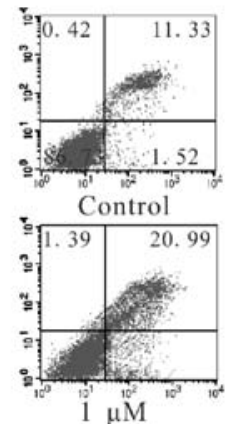

C
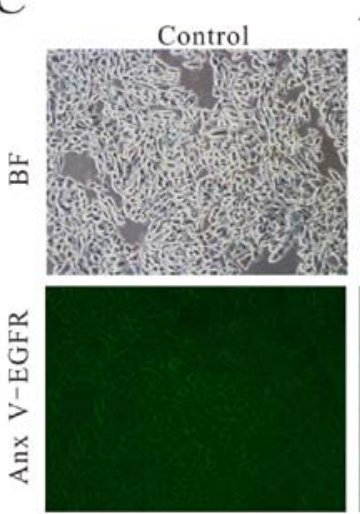
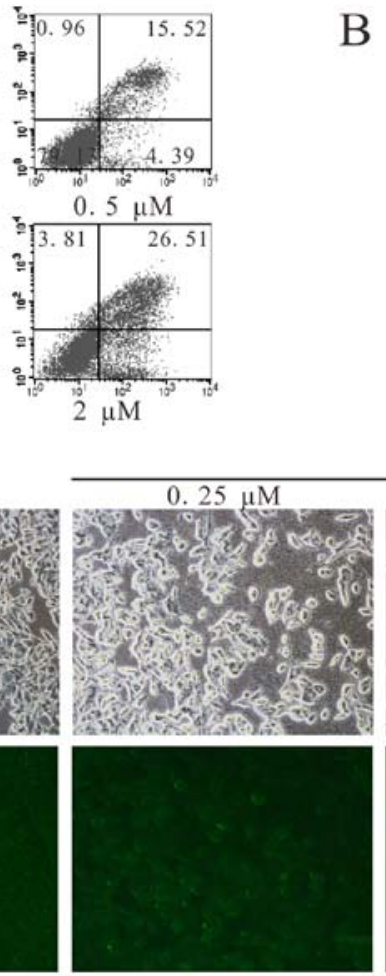

B

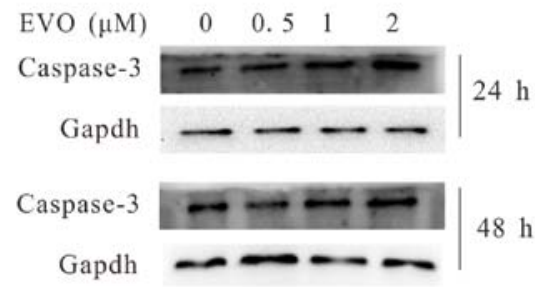

Figure 2. The apoptosis-prompting effect of EVO in human OS cells. (A) FACS analysis indicating the apoptosis induced by EVO. Cells were seeded in 6-well plates and treated with the indicated concentrations of EVO or solvent for $48 \mathrm{~h}$. Cells were then collected and stained for FACS. (B) Western blot analysis indicating the protein level of caspase-3. Cells were seeded in 6-well plates and treated with the indicated concentrations of EVO or solvent for 24 or $48 \mathrm{~h}$. Cells were then harvested and subjected to western blot analysis using an antibody against caspase-3. GAPDH was used as the loading control. (C) Annexin V-EGFP staining results indicating the apoptosis induced by EVO. 143B cells were seeded in 24-well plates and treated with EVO at the indicated concentrations for $48 \mathrm{~h}$. Cells were washed with PBS twice, and incubated with binding buffer (BF) and Annexin V-EGFP in each well for 5 min, followed by washing with PBS twice. Green fluorescent protein signal was detected under a fluorescence microscope. OS, osteosarcoma; EVO, evodiamine.

A
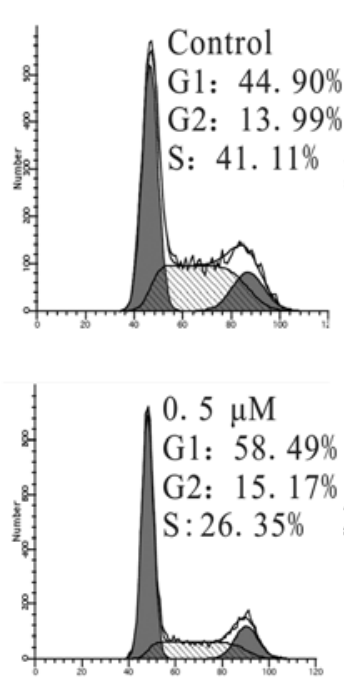
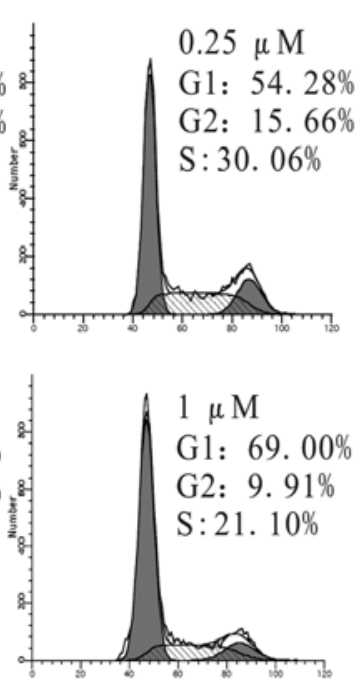

$\mathrm{B}$
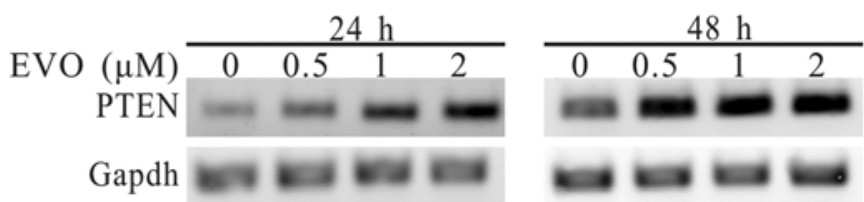

C
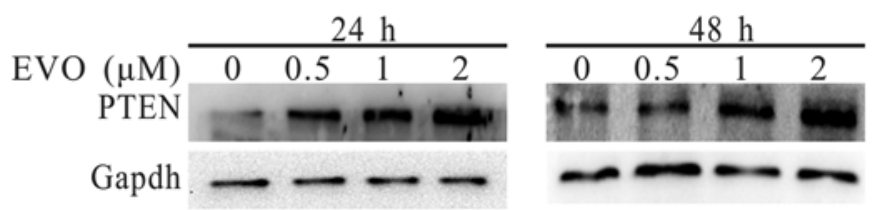

Figure 3. PTEN is involved in the anti-proliferative effect of EVO in human OS cells. (A) Cell cycle analysis showed that EVO induced cell cycle arrest at the G1 phase in the 143B OS cells. (B) EVO upregulated the mRNA expression of PTEN. 143B cells were treated with the indicated concentrations of EVO or solvent. Total RNA was extracted at the indicated time points, followed by RT-PCR assay as described in Materials and methods. (C) Western blot results indicating the effect of EVO on the protein level of PTEN. 143B cells were seeded in 6-well plates and treated with the indicated concentrations of EVO or solvent for 24 or $48 \mathrm{~h}$ and then cells were harvested for western blot analysis. GAPDH was used as the loading control and each assay was performed in triplicate. OS, osteosarcoma; EVO, evodiamine; PTEN, phosphatase and tensin homolog.

dependently suppressed the expression of PTEN in the treatment group compared to the control. To detect whether these characteristics are linked with the cell cycle arrest, we analyzed the cell cycle data in the presence of EVO and/or solvent, and found that the percentage of cells in the G1 phase was significantly increased (Fig. 3A) compared to this percentage in the 

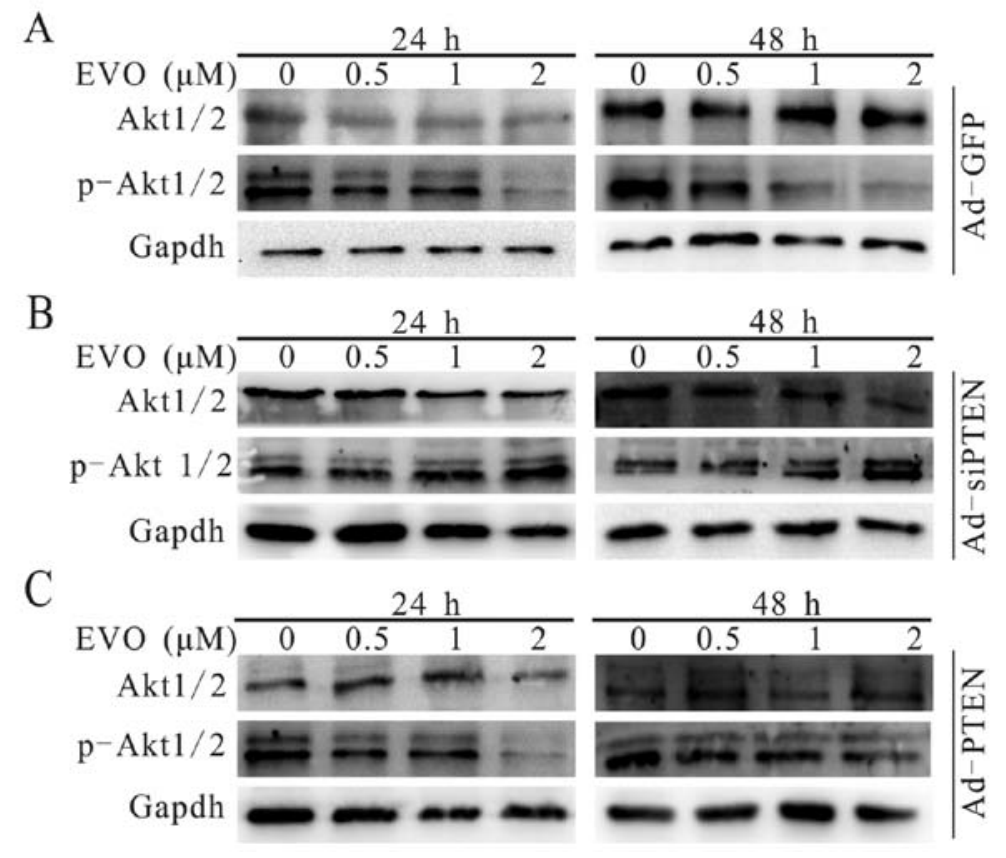

Figure 4. EVO inhibits PI3K/Akt signaling in 143B cells. (A) EVO decreased the phosphorylation of Akt1/2. 143B cells were treated with the indicated concentrations of EVO or solvent, combined with Ad-GFP. Total protein was harvested at the indicated time-points, followed by western blot analysis as described in Materials and methods. (B) Knockdown PTEN reversed the inhibition of PI3K/Akt signaling induced by EVO. 143B cells were treated with the indicated concentrations of EVO or solvent, combined with recombinant adenovirus Ad-siPTEN. Total protein was harvested at the indicated time-points, followed by western blot analysis as described in Materials and methods. GAPDH was used as the loading control. Each condition was conducted in triplicate. (C) Exogenous expression of PTEN enhanced the inhibition of PI3K/Akt signaling induced by EVO. 143B cells were treated with the indicated concentrations of EVO or solvent, combined with exogenous expression of PTEN (mediated by Ad-PTEN). Total protein was harvested at the indicated time-points, followed by western blot analysis as described in Materials and methods. OS, osteosarcoma; EVO, evodiamine; PTEN, phosphatase and tensin homolog.

control. Through further investigation, we also found that overexpression of PTEN potentiated the anti-proliferative effect of EVO, while knockdown of PTEN attenuated this effect of EVO in the 143B cells (Fig. 5A and B). Furthermore, PTEN inhibitor (VO-OHpic) reversed the anti-proliferative effect of EVO in the 143B cells (Fig. 5C and D). Based on these results, PTEN is thought to be a key gene involved in the anti-proliferative effect by EVO on human OS cells.

Evodiamine attenuates PI3K/Akt signaling transduction in 143 B cells via upregulation of PTEN. The PI3K/Akt signaling pathway, which is involved in many physiological and pathological processes of tumors, is consistently upregulated in various malignant diseases $(35,36)$. We explored whether PI3K/Akt signaling transduction participates in the EVO-induced inhibition of proliferation in the $143 \mathrm{~B}$ cells. The results demonstrated that EVO downregulated the phosphorylation level of Akt1/2 in a concentration-dependent manner (Fig. 4A). The phosphorylation level of $\mathrm{Akt} 1 / 2$ was further decreased when the 143B cells were treated with a combination of EVO and exogenous expression of PTEN (Fig. 4C). Notably, the effect of EVO on the phosphorylation of Akt1/2 was reversed following knockdown of PTEN in the 143B cells (Fig. 4B). These results indicate that PI3K/Akt signaling transduction was attenuated by PTEN during the the EVO-induced inhibition of 143B cell proliferation.

Evodiamine inhibits human osteosarcoma growth in a xenograft tumor model. The results of the in vitro experiments demonstrated the anti-osteosarcoma effects of EVO and the corresponding molecular mechanisms. We estimated the anti-osteosarcoma effects of EVO with an in vivo experiment. To construct the xenograft tumor model, 143B cells were injected into the backs of athymic nude mice by subcutaneous injection.

Three days after the injection, the athymic nude mice were treated with different doses of EVO $(20$ and $50 \mathrm{mg} / \mathrm{kg}$ ) or solvent via intragastric administration once a day for four weeks. The result revealed that the treatment groups exhibited significant suppression of tumor growth in a dose-dependent manner, compared to the control group (Fig. 6A). Hematoxylin and eosin (H\&E) staining results showed that the EVO-treated groups exhibited a decreased cellularity in the tumor masses. The H\&E histologic evaluation confirmed that the EVO-treated groups showed more necrotic cells than that in the control group (Fig. 6C). In addition, we also determined the expression of PCNA (Fig. 6B), and the results revealed that $\mathrm{EVO}$ significantly inhibited the proliferation of 143B cells in the EVO treatment group. Thus, these in vivo experimental results further demonstrated that EVO effectively inhibits OS growth.

\section{Discussion}

OS is the most frequent cancer of the bone (37). OS has a bimodal distribution; the first presentation may occur in adolescents and the second occurence is in the elderly. To date, combination therapy of multi-antitumor drugs has been 

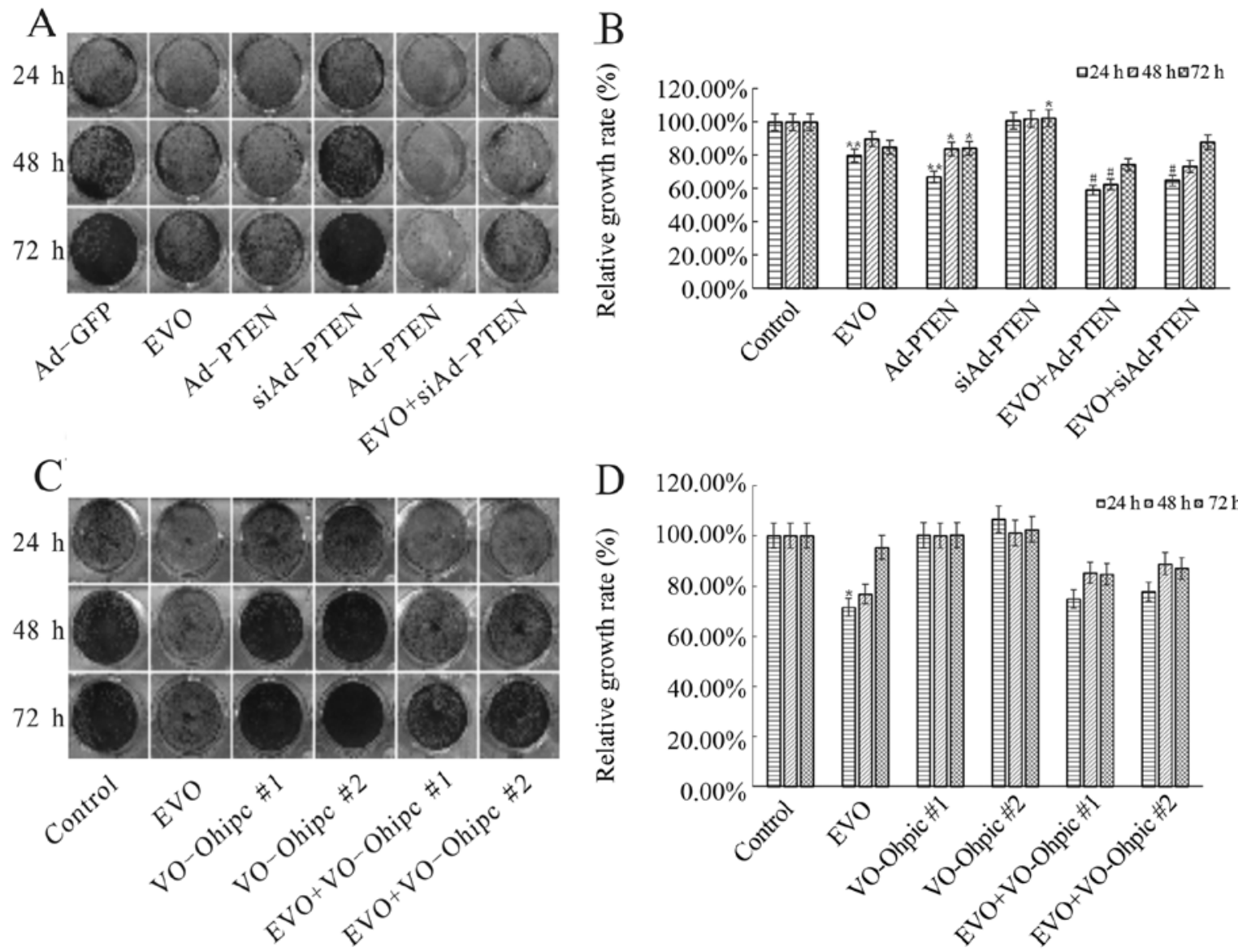

Figure 5. EVO targets PI3K/Akt signaling resulting in the inhibitory effect on human OS cell proliferation. (A and B) The effect of PTEN on the inhibition of proliferation of 143B cells mediated by EVO. 143B cells were seeded in 24-well plates and infected with Ad-PTEN or Ad-siPTEN in the presence or absence of $1 \mu \mathrm{M} \mathrm{EVO}$. At 24, 48 and $72 \mathrm{~h}$ after treatment, the cells were stained with crystal violet and the growth rate was quantified. The assay was performed in triplicate. ${ }^{*} \mathrm{P}<0.05$, compared with the control; ${ }^{* *} \mathrm{P}<0.01$, compared with the control; ${ }^{*} \mathrm{P}<0.05$, compared with EVO. (C and D) The effect of specific PTEN inhibitor (VO-Ohipc) on the proliferation-reducing effects of EVO on 143B cells. The 143B cells were seeded in 24-well plates and treated with the indicated concentrations of VO-Ohipc (\#1, $2 \mathrm{nM} ; \# 2,4 \mathrm{nM}$ ) in the presence or absence of $1 \mu \mathrm{M}$ EVO. At 24, 48 and $72 \mathrm{~h}$ after treatment, the cells were stained with crystal violet and the growth rate was quantified. The assay was performed in triplicate. ${ }^{*} \mathrm{P}<0.05$, compared with the control; ${ }^{* *} \mathrm{P}<0.01$, compared with the control; "P<0.05, compared with EVO. OS, osteosarcoma; EVO, evodiamine; PTEN, phosphatase and tensin homolog.

introduced for OS treatment for the past 40 years, yet $\sim 80 \%$ of patients with local early stage tumors progress to a metastatic status $(2,38)$, and recurrent and/or metastatic OS tumors are extremely invasive and even resistant to traditional antitumor strategies (2). Therefore, identification of a novel treatment modality with more efficacy for OS patients is vital.

EVO, (Chinese name, Wu-Chu-Yu), a quinolone alkaloid, is the essential component extracted from the fruit of Evodia rutaecarpa $(39,40)$. The role of EVO in the inhibition of tumor cell proliferation has been well-documented in a number of studies (13-20), while our knowledge of the antitumor effects of EVO on OS cells remains rather sparse. To the best of our knowledge, this is the first study to explore whether EVO possesses anticancer activities in OS cells, and the possible molecular mechanism involved. Based on the data presented in the present study, we found that EVO significantly inhibited the proliferation and induced the apoptosis in 143B cells in a time- and concentration-dependent manner, even at a concentration of $0.5 \mu \mathrm{M}$ (Fig. 1A and B). More importantly, it has been reported that EVO exhibits low cytotoxicity to human peripheral blood cells (41) and increasing evidence suggests that EVO has strong specificity to tumor cells. Mechanistically, our results also showed that overexpression of PTEN is responsible for the anti-proliferation effect of EVO on 143B OS cells. Furthermore, PI3K/Akt signaling transduction is inhibited via EVO-induced upregulation of PTEN.

According to previous research, EVO was found to strongly inhibit tumor progression by reducing the proliferation rate and inducing apoptosis in a variety of tumor cells (13-20). A series of analyses attempted to explain the underlying molecular mechanisms. A large number of trials have shown that EVO can cause cell cycle arrest at the $\mathrm{G} 2 / \mathrm{M}$ phase in a majority of cancer cells (42), yet research on the cell cycle regulation by EVO in OS cells has not been carried out. Kan et al found that EVO activated the Cdc2/cyclin B complex to regulate cycle cycle arrest $(\mathrm{G} 2 / \mathrm{M})$ in human prostate cancer cell lines DU145 and PC3 (18). Conversely, EVO developed atypical apoptosis in murine fibrosarcoma L929 cells by cell cycle arrest at the G0/G1 phase (15). Our research obtained similar results (Fig. 3A), suggesting that the cell cycle regulation by EVO in 143B cells may be different from that in other types of tumor cells. In addition, Takada et al found 
A

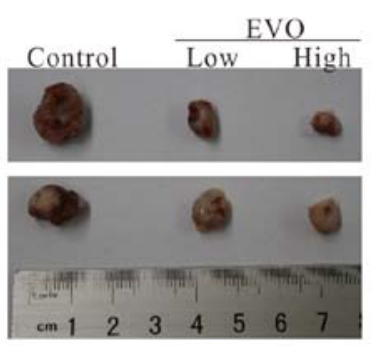

$\mathrm{C}$
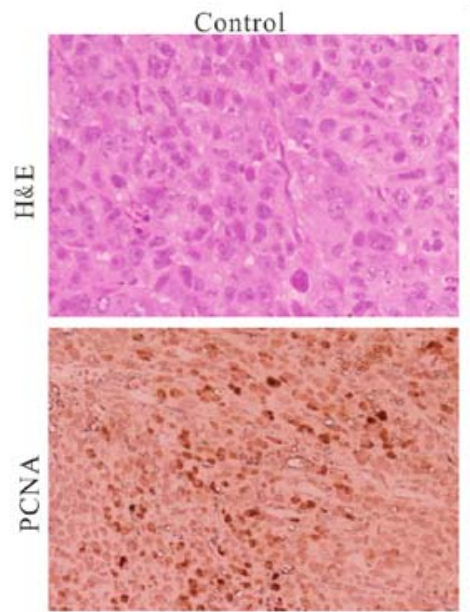

B

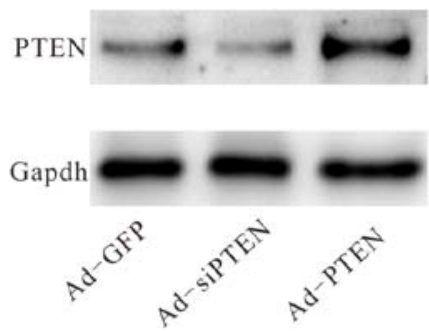

EVO

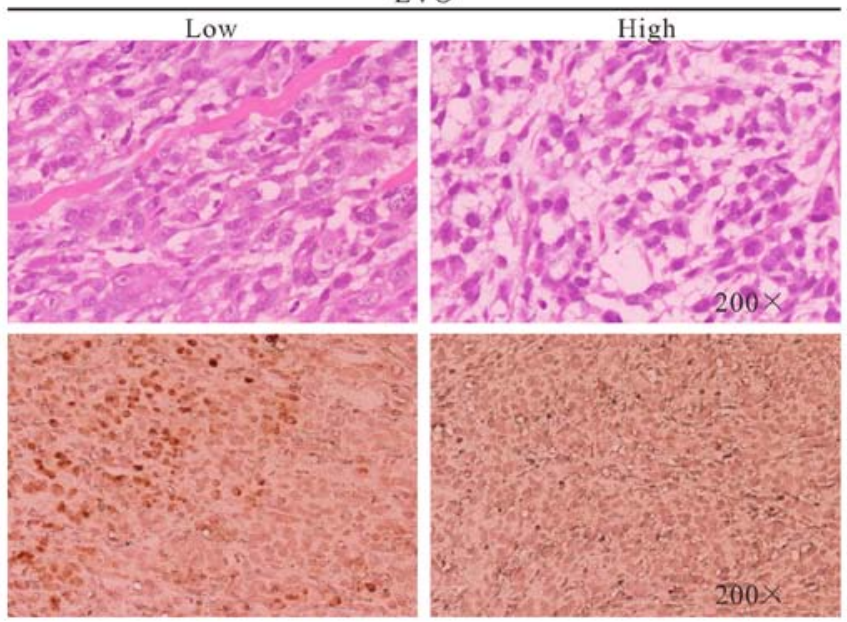

Figure 6. EVO inhibits xenograft tumor growth of OS. (A) The tumor growth-inhibitory effect of EVO on xenograft tumor growth. After the final treatment, tumor samples were retrieved from the OS tumor animal model. (B) The effect of recombinant adenovirus on the expression of PTEN. 143B cells were seeded in a 6-well plate and infected with Ad-GFP, Ad-PTEN and Ad-siPTEN. After $24 \mathrm{~h}$, total protein was harvested for western blot analysis to measure the expression of PTEN. Each condition was conducted in triplicate. (C) Histologic and immunohistochemical staining of EVO-treated OS cell tumors. Tumor samples derived from 143B xenografts were retrieved, fixed and paraffin-embedded. Sections were used for H\&E staining. Deparaffinized slides were stained with antibody against PCNA. Representative results are shown. OS, osteosarcoma; EVO, evodiamine; PTEN, phosphatase and tensin homolog; H\&E, hematoxylin and eosin.

that EVO exerts apoptotic activity by regulating $\mathrm{NF}-\kappa \mathrm{B}$ activation, resulting in the inhibition of $\mathrm{NF}-\kappa \mathrm{B}-$ controlled downstream genes, including cyclin D1, c-Myc, survivin, $\mathrm{X}$ chromosome-linked IAP (XIAP), Bcl-2 and Bcl-X1 (43). Numerous experiments have demonstrated that EVO activated initiator caspases (caspase-8 and 9) and/or effector caspases (caspase-3) to induce the apoptosis in a variety of tumor cell lines including human colorectal carcinoma COLO205 and HT-29 cells (44) and human thyroid cancer cell line ARO (45). In addition, another study showed that EVO promoted translocation of apoptosis-inducing factor (AIF) into the nucleus of human leukemia U937 cells (46). Collectively, EVO was found to induce the apoptosis of many tumor cell lines via caspase-dependent and caspase-independent signaling pathways (47). Moreover, Liu et al proved that EVO-mediated autophagy leading to increased apoptosis and reduction in cell viability was via a calcium-JNK signaling pathway (48). Further study suggested that the downregulation of PI3K/Akt/ caspase and Fas-L/NF- $\kappa \mathrm{B}$ signaling pathways may be responsible for the apoptosis of A375-S cells induced by EVO (49). Although a number of studies have analyzed the possible molecular mechanisms by which EVO exhibits inhibitory action on tumorigenesis in various cancer cells, the inhibitory effect of EVO on OS cells and the possible molecular mecha- nism of the signaling transduction invovled in the anticancer activity of EVO remain unclear.

PTEN, a tumor suppressor, is commonly mutated in the majority of human epithelial cell-derived tumors (50). On account of its phospholipid phosphatase characteristic, PTEN regulates various cellular processes and potentially affects the transduction of many other signaling pathways. PTEN is able to catalyze 3'-phosphate on PIP3 to form PIP2, thereby, $\mathrm{PI} 3 \mathrm{~K} / \mathrm{Akt}$ signaling transduction is directly abrogated by PTEN (28). Despite the high aberration rate of PTEN in many types of cancers, it is controversial that PTEN mutations are linked with OS tumorigenesis. For example, the biallelic and monoallelic mutation rate of PTEN in OS samples was found to reach $\sim 15$ and $33 \%$, respectively (51). The present results indicated that both the protein and gene levels of PTEN were increased concentration-dependently in the EVO-induced 143B cells. Exogenous PTEN strengthened the effect of EVO in 143B cells, while VO-OHpic treatment or knockdown of PTEN reversed the inhibitory effect on proliferation caused by EVO. Further research showed that EVO upregulated the expression of PTEN through the deactivation of PI3K/Akt signaling transduction by the reduction of phosphorylated Akt1/2 in 143B cells. The in vivo experiment results showed that EVO inhibited tumorgenesis. Collectively, PTEN/ 
PI3K/Akt signaling was found to participate in the inhibition of proliferation of human OS 143B cells by EVO.

The present results suggest that EVO may be a promising antitumor strategy against human OS. Further studies are warranted to elucidate additional targets of EVO and the specific molecular mechanisms of the anticancer activity of EVO. In addition, a number of pre-clinical assessments should be carried out for the analysis of drug safety.

\section{Acknowledgements}

We would like to thank Dr T.C. He (University of Chicago Medical Center, USA) for generously providing all recombinant adenoviruses.

\section{References}

1. Graudal N, Hubeck-Graudal T, Tarp S, Christensen R and Jurgens G: Effect of combination therapy on joint destruction in rheumatoid arthritis: A network meta-analysis of randomized controlled trials. PLoS One 9: e106408, 2014.

2. Tang N, Song WX, Luo J, Haydon RC and He TC: Osteosarcoma development and stem cell differentiation. Clin Orthop Relat Res 466: 2114-2130, 2008.

3. Mirabello L, Troisi RJ and Savage SA: Osteosarcoma incidence and survival rates from 1973 to 2004: Data from the Surveillance, Epidemiology, and End Results Program. Cancer 115: 1531-1543, 2009.

4. Marina N, Gebhardt M, Teot L and Gorlick R: Biology and therapeutic advances for pediatric osteosarcoma. Oncologist 9: 422-441, 2004.

5. Price $\mathrm{CH}$ : Osteogenic sarcoma; an analysis of the age and sex incidence. Br J Cancer 9: 558-574, 1955.

6. Weinfeld MS and Dudley HR Jr: Osteogenic sarcoma. A follow-up study of the ninety-four cases observed at the Massachusetts General Hospital from 1920 to 1960. J Bone Joint Surg Am 44-A: 269-276, 1962.

7. Dahlin DC and Coventry MB: Osteogenic sarcoma. A study of six hundred cases. J Bone Joint Surg Am 49: 101-110, 1967.

8. Ando K, Heymann MF, Stresing V, Mori K, Redini F and Heymann D: Current therapeutic strategies and novel approaches in osteosarcoma. Cancers (Basel) 5: 591-616, 2013.

9. Anderson PM and Pearson M: Novel therapeutic approaches in pediatric and young adult sarcomas. Curr Oncol Rep 8: 310-315, 2006.

10. Lin H, Tsai SC, Chen JJ, Chiao YC, Wang SW, Wang GJ, Chen CF and Wang PS: Effects of evodiamine on the secretion of testosterone in rat testicular interstitial cells. Metabolism 48: 1532-1535, 1999.

11. Chiou WF, Sung YJ, Liao JF, Shum AY and Chen CF: Inhibitory effect of dehydroevodiamine and evodiamine on nitric oxide production in cultured murine macrophages. J Nat Prod 60 708-711, 1997.

12. Kobayashi Y: The nociceptive and anti-nociceptive effects of evodiamine from fruits of Evodia rutaecarpa in mice. Planta Med 69: 425-428, 2003.

13. King CL, Kong YC, Wong NS, Yeung HW, Fong HH and Sankawa U: Uterotonic effect of Evodia rutaecarpa alkaloids. J Nat Prod 43: 577-582, 1980.

14. Hong JY, Park SH, Min HY, Park HJ and Lee SK: Anti-proliferative effects of evodiamine in human lung cancer cells. J Cancer Prev 19: 7-13, 2014.

15. Zhang Y, Zhang QH, Wu LJ, Tashiro S, Onodera S and Ikejima T: Atypical apoptosis in L929 cells induced by evodiamine isolated from Evodia rutaecarpa. J Asian Nat Prod Res 6: $19-27,2004$

16. Huang YC, Guh JH and Teng CM: Induction of mitotic arrest and apoptosis by evodiamine in human leukemic T-lymphocytes. Life Sci 75: 35-49, 2004.

17. Fei XF, Wang BX, Li TJ, Tashiro S, Minami M, Xing DJ and Ikejima T: Evodiamine, a constituent of Evodiae Fructus, induces anti-proliferating effects in tumor cells. Cancer Sci 94: 92-98, 2003
18. Kan SF, Yu CH, Pu HF, Hsu JM, Chen MJ and Wang PS: Anti-proliferative effects of evodiamine on human prostate cancer cell lines DU145 and PC3. J Cell Biochem 101: 44-56, 2007.

19. Kan SF, Huang WJ, Lin LC and Wang PS: Inhibitory effects of evodiamine on the growth of human prostate cancer cell line LNCaP. Int J Cancer 110: 641-651, 2004.

20. Huang DM, Guh JH, Huang YT, Chueh SC, Chiang PC and Teng CM: Induction of mitotic arrest and apoptosis in human prostate cancer pc-3 cells by evodiamine. J Urol 173: 256-261, 2005.

21. Yang J, Cai X, Lu W, Hu C, Xu X, Yu Q and Cao P: Evodiamine inhibits STAT3 signaling by inducing phosphatase shatterproof 1 in hepatocellular carcinoma cells. Cancer Lett 328: 243-251, 2013.

22. Porta C, Paglino $\mathrm{C}$ and Mosca A: Targeting PI3K/Akt/mTOR signaling in cancer. Front Oncol 4: 64, 2014.

23. Zhang S and Yu D: PI(3)king apart PTEN's role in cancer. Clin Cancer Res 16: 4325-4330, 2010

24. Mahimainathan L and Choudhury GG: Inactivation of platelet-derived growth factor receptor by the tumor suppressor PTEN provides a novel mechanism of action of the phosphatase. J Biol Chem 279: 15258-15268, 2004.

25. Yoshimoto M, Cunha IW, Coudry RA, Fonseca FP, Torres CH, Soares FA and Squire JA: FISH analysis of 107 prostate cancers shows that PTEN genomic deletion is associated with poor clinical outcome. Br J Cancer 97: 678-685, 2007.

26. Ishii N, Maier D, Merlo A, Tada M, Sawamura Y, Diserens AC and Van Meir EG: Frequent co-alterations of TP53, p16/CDKN2A, p14ARF, PTEN tumor suppressor genes in human glioma cell lines. Brain Pathol 9: 469-479, 1999.

27. Maehama T and Dixon JE: The tumor suppressor,PTEN/MMAC1, dephosphorylates the lipid second messenger, phosphatidylinositol 3,4,5-trisphosphate. J Biol Chem 273: 13375-13378, 1998.

28. Franke TF, Kaplan DR, Cantley LC and Toker A: Direct regulation of the Akt proto-oncogene product by phosphatidylinositol-3,4-bisphosphate. Science 275: 665-668, 1997.

29. He BC, Chen L, Zuo GW, Zhang W, Bi Y, Huang J, Wang Y, Jiang W, Luo Q, Shi Q, et al: Synergistic antitumor effect of the activated PPARgamma and retinoid receptors on human osteosarcoma. Clin Cancer Res 16: 2235-2245, 2010.

30. He TC, Zhou S, da Costa LT, Yu J, Kinzler KW and Vogelstein B: A simplified system for generating recombinant adenoviruses. Proc Natl Acad Sci USA 95: 2509-2514, 1998.

31. He BC, Gao JL, Luo X, Luo J, Shen J, Wang L, Zhou Q, Wang YT, Luu HH, Haydon RC, et al: Ginsenoside Rg3 inhibits colorectal tumor growth through the downregulation of Wnt/ss-catenin signaling. Int J Oncol 38: 437-445, 2011.

32. Strzalka W and Ziemienowicz A: Proliferating cell nuclear antigen (PCNA): A key factor in DNA replication and cell cycle regulation. Ann Bot 107: 1127-1140, 2011.

33. Singha PK, Pandeswara S, Geng H, Lan R, Venkatachalam MA and Saikumar P: TGF-beta induced TMEPAI/PMEPA1 inhibits canonical Smad signaling through R-Smad sequestration and promotes non-canonical PI3K/Akt signaling by reducing PTEN in triple negative breast cancer. Genes Cancer 5: 320-336, 2014.

34. Marques RB, Aghai A, de Ridder CM, Stuurman D, Hoeben S, Boer A, Ellston RP, Barry ST, Davies BR, Trapman J, et al: High efficacy of combination therapy using PI3K/AKT inhibitors with androgen deprivation in prostate cancer preclinical models. Eur Urol: Sep 11, 2014 (Epub ahead of print) doi: 10.1016/j. eururo.2014.08.053.

35. Wang J, Chu ES, Chen HY, Man K, Go MY, Huang XR, Lan HY, Sung JJ and Yu J: microRNA-29b prevents liver fibrosis by attenuating hepatic stellate cell activation and inducing apoptosis through targeting PI3K/AKT pathway. Oncotarget 6: 7325-7338, 2014.

36. Yu P, Ye L, Wang H, Du G, Zhang J, Zhang J and Tian J: NSK-01105 inhibits proliferation and induces apoptosis of prostate cancer cells by blocking the Raf/MEK/ERK and PI3K/Akt/mTOR signal pathways. Tumour Biol 36: 2143-2153, 2015.

37. Cormier JN and Pollock RE: Soft tissue sarcomas. CA Cancer J Clin 54: 94-109, 2004.

38. Gorlick R, Anderson P, Andrulis I, Arndt C, Beardsley GP, Bernstein M, Bridge J, Cheung NK, Dome JS, Ebb D, et al: Biology of childhood osteogenic sarcoma and potential targets for therapeutic development: meeting summary. Clin Cancer Res 9: 5442-5453, 2003. 
39. Wang L, Hu CP, Deng PY, Shen SS, Zhu HQ, Ding JS, Tan GS and Li YJ: The protective effects of rutaecarpine on gastric mucosa injury in rats. Planta Med 71: 416-419, 2005.

40. Yu X, Wu DZ, Yuan JY, Zhang RR and Hu ZB: Gastroprotective effect of fructus evodiae water extract on ethanol-induced gastric lesions in rats. Am J Chin Med 34: 1027-1035, 2006.

41. Liao CH, Pan SL, Guh JH, Chang YL, Pai HC, Lin CH and Teng CM: Antitumor mechanism of evodiamine, a constituent from Chinese herb Evodiae fructus, in human multiple-drug resistant breast cancer NCI/ADR-RES cells in vitro and in vivo. Carcinogenesis 26: 968-975, 2005.

42. Jiang $\mathrm{J}$ and $\mathrm{Hu} \mathrm{C}$ : Evodiamine: a novel anti-cancer alkaloid from Evodia rutaecarpa. Molecules 14: 1852-1859, 2009.

43. Takada Y, Kobayashi Y and Aggarwal BB: Evodiamine abolishes constitutive and inducible NF-kappaB activation by inhibiting IkappaBalpha kinase activation, thereby suppressing NF-kappaB-regulated antiapoptotic and metastatic gene expression, upregulating apoptosis, and inhibiting invasion. J Biol Chem 280: 17203-17212, 2005.

44. Chien CC, Wu MS, Shen SC, Ko CH, Chen CH, Yang LL and Chen YC: Activation of JNK contributes to evodiamine-induced apoptosis and $\mathrm{G} 2 / \mathrm{M}$ arrest in human colorectal carcinoma cells: a structure-activity study of evodiamine. PLoS One 9: e99729, 2014.

45. Chen MC, Yu CH, Wang SW, Pu HF, Kan SF, Lin LC, Chi CW, Ho LL, Lee CH and Wang PS: Anti-proliferative effects of evodiamine on human thyroid cancer cell line ARO. J Cell Biochem 110: 1495-1503, 2010.
46. Lee TJ, Kim EJ, Kim S, Jung EM, Park JW, Jeong SH, Park SE, Yoo YH and Kwon TK: Caspase-dependent and caspase-independent apoptosis induced by evodiamine in human leukemic U937 cells. Mol Cancer Ther 5: 2398-2407, 2006.

47. Yu H, Jin H, Gong W, Wang Z and Liang H: Pharmacological actions of multi-target-directed evodiamine. Molecules 18: 1826-1843, 2013.

48. Liu AJ, Wang SH, Chen KC, Kuei HP, Shih YL, Hou SY, Chiu WT, Hsiao SH and Shih CM: Evodiamine, a plant alkaloid, induces calcium/JNK-mediated autophagy and calcium/mitochondria-mediated apoptosis in human glioblastoma cells. Chem Biol Interact 205: 20-28, 2013.

49. Wang C, Li S and Wang MW: Evodiamine-induced human melanoma A375-S2 cell death was mediated by PI3K/Akt/caspase and Fas-L/NF-kappaB signaling pathways and augmented by ubiquitin-proteasome inhibition. Toxicol In Vitro 24: 898-904, 2010.

50. Ali IU, Schriml LM and Dean M: Mutational spectra of PTEN/MMAC1 gene: a tumor suppressor with lipid phosphatase activity. J Natl Cancer Inst 91: 1922-1932, 1999.

51. Freeman SS, Allen SW, Ganti R, Wu J, Ma J, Su X, Neale G, Dome JS, Daw NC and Khoury JD: Copy number gains in EGFR and copy number losses in PTEN are common events in osteosarcoma tumors. Cancer 113: 1453-1461, 2008. 